\title{
Tuberculose primaire de la glande parotide : à propos d'un cas
}

\section{Primary tuberculosis of the parotid gland: a case report}

\section{MOTS-CLEFS :}

- Tuberculose, parotide

\section{KEYWORDS:}

- Tuberculosis, parotid gland
AOS 2015;271:18-20 DOI: $10.1051 /$ aos/2015103 (C) EDP Sciences 2015

\section{Résumé}

La tuberculose primaire de la glande parotide est une entité clinique rare. Le diagnostic est difficile, puisqu'il n'existe aucun signe clinique, radiologique ou biologique spécifique de cette affection. Seules les données bactériologiques et anatomopathologiques permettent d'affirmer le diagnostic. La prise en charge thérapeutique repose sur la prescription d'un traitement antituberculeux au long cours, avec une évolution généralement favorable.

L'objectif de ce travail est de rapporter un nouveau cas de tuberculose primaire de la glande parotide chez une jeune femme, et d'insister sur les difficultés diagnostiques de cette affection.

\section{Abstract}

Tuberculosis of the parotid gland is a rare clinical entity. Diagnosis is difficult because there are no specific clinical, radiological or biological signs of the disease. Only bacteriological and histopathological findings can confirm the diagnosis. Treatment is based on antituberculous drugs and allows usually a favourable evolution.

The aim of this paper is to report on a case of primary tuberculosis of the parotid gland in a 21 -year-old female, and to stress the corresponding diagnostic pitfalls.

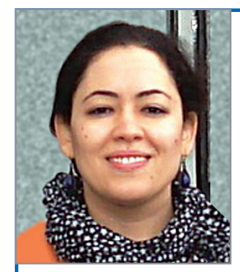

- Dounia KAMAL, Professeur assistante en stomatologie et chirurgie maxillo-faciale, service d'ORL, CHU Hassan II, Fès, Maroc.

Email : kamaldounia@hotmail.com

Ayat OUFKIR, Professeur agrégé de chirurgie plastique, service d'ORL, CHU Hassan II, Fès, Maroc.

Alae BEZZARI, Médecin résident en ORL, service d'ORL, CHU Hassan II, Fès, Maroc.

Mustapha MAAROUFI, Professeur agrégé de radiologie, service de radiologie, CHU Hassan II, Fès, Maroc.

Mohamed Nourreddine EL ALAMI, Professeur d'ORL et chef du service d'ORL, CHU Hassan II, Fès, Maroc. 


\section{INTRODUCTION}

La tuberculose est une infection granulomateuse chronique due à Mycobacterium tuberculosis ou bovis qui peut toucher tous les organes. Au niveau de la sphère ORL, l'atteinte ganglionnaire est la plus fréquente. La localisation isolée de la tuberculose au niveau des glandes salivaires, et notamment de la parotide, est extrêmement rare [1,2]. La présentation clinique est polymorphe et non spécifique.

Nous rapportons un cas de tuberculose parotidienne simulant une tumeur chez une jeune femme de 21 ans, et nous discutons les différents éléments diagnostiques, thérapeutiques et évolutifs de cette pathologie.

\section{OBSERVATION}

Une jeune femme de 21 ans, sans antécédent pathologique notable, a consulté dans notre formation pour une masse parotidienne droite évoluant depuis environ 5 mois (fig. 1). Il n'existait pas de signes cliniques associés, notamment pas de douleurs, de fièvre, d’amaigrissement, de gêne à la mastication, de trismus ou d'issue de pus en endobuccal.

La jeune femme était en bon état général et présentait une tuméfaction de la région parotidienne droite, ferme, indolore, mesurant environ $5 \mathrm{~cm}$ de grand axe, fixe. Il nexistait pas de limitation de louverture buccale, ni de paralysie faciale périphérique.

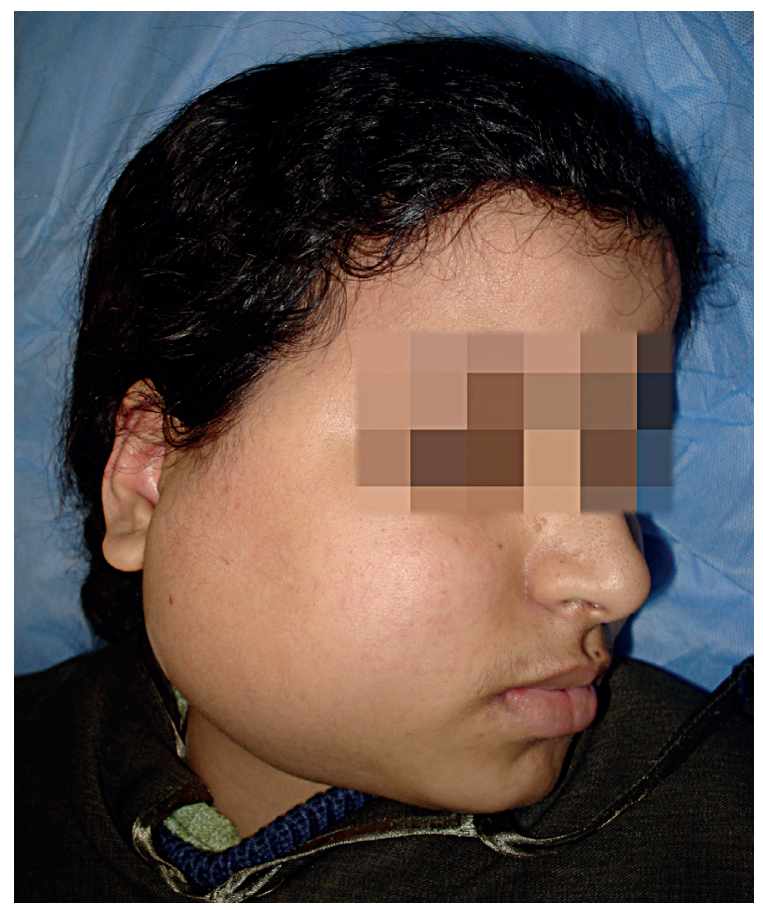

$\triangle$ Fig. 1 : photo exobuccale montrant la tuméfaction de la région parotidienne droite.
Lexamen de la cavité buccale et de l'orifice du canal de Sténon ne mettait pas en évidence d'inflammation ni d'issue de pus à ce niveau. L'examen des aires ganglionnaires cervicales permettait de palper quelques adénopathies lenticulaires homolatérales, jugulo-carotidiennes. Le reste de l'examen ORL et général était sans particularité.

Une tomodensitométrie cervico-faciale a montré une collection intraparotidienne droite, hétérogène, cernée par une coque périphérique irrégulière et rehaussée (fig. 2).

Le bilan biologique était normal, ainsi que la radiographie pulmonaire. Nous avons complété les investigations par une intradermoréaction à la tuberculine, qui était positive, mesurée à $15 \mathrm{~mm}$ de diamètre.

Au cours des explorations, lévolution a été marquée par la fistulisation spontanée de la masse au niveau cervical, avec issue de pus. Nous avons effectué un curetage biopsique à travers lorifice fistuleux, avec prélèvements bactériologiques et anatomopathologiques. L’étude histopathologique de la paroi du kyste a retrouvé un granulome épithéliogigantocellulaire avec de la nécrose caséeuse. La mise en culture du liquide de ponction sur milieu de Lowenstein-Jensen a mis en évidence un bacille acidoalcoolorésistant, nous permettant ainsi de confirmer le diagnostic de tuberculose de la glande parotide.

La jeune femme a reçu un traitement médical à base d'antituberculeux pour une durée de 6 mois. L'évolution a été favorable avec un recul de 6 mois après la fin du traitement.

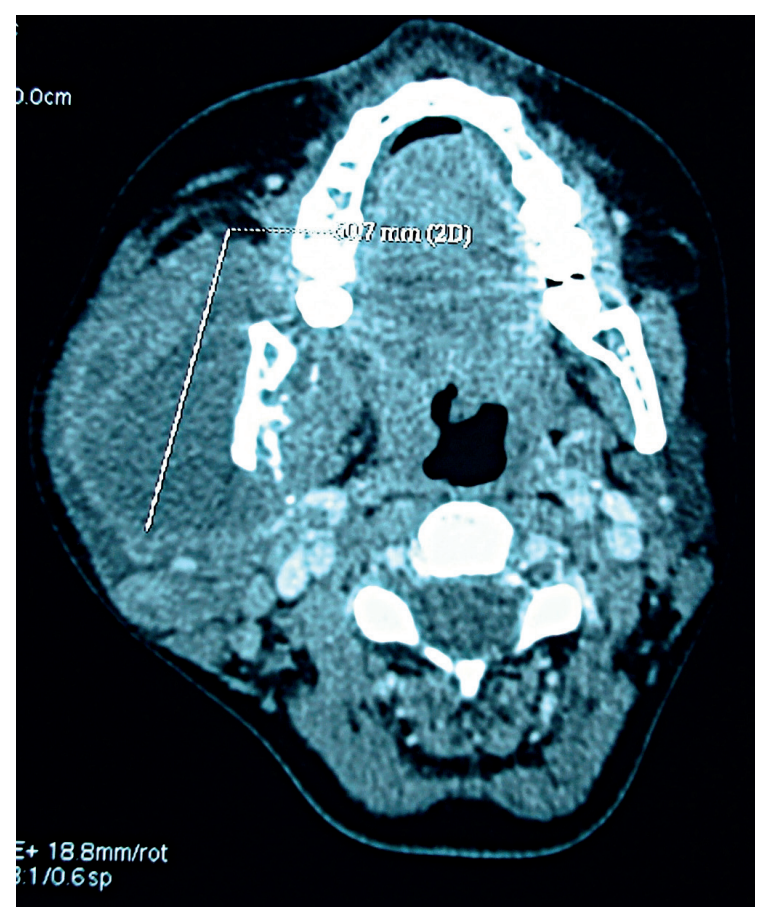

$\triangle$ Fig. 2 : coupe axiale en fenêtre parenchymateuse montrant une collection intraparotidienne droite. 


\section{DISCUSSION}

Décrite pour la première fois en 1894 par von Stubenrauch, la tuberculose parotidienne est rare. Plus de $90 \%$ des cas décrits le sont dans les pays en voie de développement [3]. Elle atteint le plus souvent le sujet jeune, entre 20 et 40 ans.

La localisation parotidienne parenchymateuse se voit le plus souvent dans le cadre d'une tuberculose disséminée, la diffusion se faisant par voie hématogène ou lymphatique à partir d'un foyer pulmonaire ou est isolée, comme pour notre patient, posant un problème de diagnostic différentiel avec la pathologie tumorale de la glande parotide. Dans ce cas, le Mycobacterium, localisé au niveau oropharyngé, passe par voie canalaire rétrograde à travers le canal de Sténon. Il faut la distinguer d'une atteinte tuberculeuse des ganglions intraparotidiens qui est beaucoup plus fréquente. Cliniquement, la tuberculose parotidienne se manifeste généralement sous forme d'une tuméfaction parotidienne unilatérale d'installation progressive, pouvant être diffuse ou nodulaire, réalisant un syndrome pseudotumoral. La paralysie faciale, le caractère douloureux et le trismus peuvent être retrouvés, mais sont plus en faveur d'un processus malin. En revanche, la présence d'une fistule cutanée est très évocatrice d'une pathologie inflammatoire. Les signes généraux d'imprégnation tuberculeuse sont rarement présents, mais doivent être recherchés et peuvent orienter le diagnostic, ainsi que l'origine géographique du patient [3]. Les données du bilan radiologique (échographie, TDM et IRM) ne permettent généralement pas de poser le diagnostic.

En effet, différents aspects très variables peuvent se voir : nodule tissulaire parotidien, sialadénite diffuse, kyste ou processus de nature liquidienne, plus ou moins associés à des adénopathies intraparotidiennes et jugulocarotidiennes. La sialographie n'est pas spécifique, tout comme la scintigraphie au technétium $99 \mathrm{~m}$ ou au gallium 67 . Une radiographie pulmonaire doit systématiquement être demandée, afin de rechercher un éventuel foyer primitif. Sur le plan biologique, on retrouve généralement un syndrome inflammatoire et l'intradermoréaction à la tuberculine n’est pas toujours positive [2-5].

Ainsi, aucun élément clinique, radiologique et biologique ne permet d'affirmer le diagnostic de tuberculose parotidienne. De ce fait, le diagnostic différentiel des formes diffuses envisage surtout la parotidite lithiasique ou un carcinome, la forme circonscrite évoquant plutôt un kyste, une adénite ou une tumeur mixte.

Le challenge du praticien est donc de faire le diagnostic avant l'exploration chirurgicale et l'examen histologique de la pièce de parotidectomie.
La cytoponction à l'aiguille fine avec mise en culture du liquide de ponction peut être utile, mais n'a de valeur que si elle est positive. Elle a une spécificité de $81 \%$ et une sensibilité de $94 \%$ [5]. Actuellement, la plupart des auteurs recommandent les techniques d'amplification génique par PCR (Polymerase Chain Reaction) après culture du broyat cellulaire glandulaire permettant d'augmenter les résultats positifs de la cytoponction [6]. D’autres proposent un test thérapeutique aux antituberculeux lorsque le diagnostic est fortement suspecté $[3,4]$.

Cependant, dans certains cas, seul l'examen histologique d'une adénopathie satellite ou parfois même d'une parotidectomie permettra de poser le diagnostic en mettant en évidence le classique granulome épithéliogigantocellulaire avec nécrose caséeuse [5].

La prise en charge thérapeutique est essentiellement médicale.

La parotidectomie exofaciale a longtemps été recommandée, à visée diagnostique et pour permettre une meilleure diffusion des antituberculeux. Elle est actuellement abandonnée par tous les auteurs du fait de sa morbidité non négligeable et de l'efficacité du traitement médical seul. Il repose sur la prescription d'antituberculeux au long cours, généralement pendant six à neuf mois, permettant une disparition rapide du syndrome tumoral parotidien $[3,5,6]$.
[1] Chatterjee A, Varman M, Quinlan TW. Parotid abscess caused by Mycobacterium tuberculosis. Pediatr Infect Dis J 2001;20(9):912-4.

[2] Kim YH, Jeong WJ, Jung KY, Sung MW, Kim KH, Kim CS. Diagnosis of major salivary gland tuberculosis: experience of eight cases and review of the literature. Acta Otolaryngol 2005;125(12):1318-22.

[3] Suleiman AM. Tuberculous parotitis: report of three cases. Br J Oral Maxillofac Surg 2001;39(4):320-3.

[4] Sethi A, Sareen D, Sabherwal A, Malhotra V, Primary parotid tuberculosis: varied clinical presentations. Oral Dis 2006;12(2):213-5.

[5] Handa U, Kumar S, Punia RS, Mohan H, Abrol R, Saini V. Tuberculous parotitis: a series of five cases diagnosed on fine needle aspiration cytology. J Laryngol Otol 2001;115(3):235-7.

[6] Kontopoulou T et al. Tuberculosis of the parotid gland: case report and literature review. Med Mal Infect 2004;34(10):488-90. 